\title{
Die soziale Integration von Schülerinnen und Schülern mit sozialer Unsicherheit
}

\author{
Simone Weber (iD, Philipp Nicolay und Christian Huber \\ Bergische Universität Wuppertal, Deutschland
}

\begin{abstract}
Zusammenfassung: Der vorliegende Beitrag untersucht den Zusammenhang zwischen sozialer Integration und sozialer Unsicherheit. Ausgehend von dem mehrdimensionalen Modell sozialer Integration (Koster, Nakken, Pijl \& van Houten, 2009) wird diese differenziert anhand der Dimensionen Beziehungen, Kontakte, Akzeptanz durch Mitschülerinnen und Mitschüler und eigene Wahrnehmung analysiert. Hierfür wurden Daten von N = 617 Kindern aus insgesamt 28 Schulklassen der dritten und vierten Jahrgangsstufe geprüft. Die Ergebnisse der Mehrebenenanalysen zeigen einen signifikanten negativen Zusammenhang zwischen sozialer Unsicherheit und sozialer Integration in drei der vier Dimensionen (Beziehungen, eigene Wahrnehmung und Akzeptanz). Die Ergebnisse für die einzelnen Dimensionen werden diskutiert und es werden Implikationen für die Forschung und pädagogische Praxis abgeleitet.
\end{abstract}

Schlüsselwörter: Soziale Unsicherheit, soziale Integration, Primarstufe

The social integration of students with social insecurity

Abstract: This paper analyses the relationship between social integration and social insecurity. Based on the multidimensional model of social integration (Koster et al., 2009), the dimensions relationships, contacts, acceptance by classmates and self-perception are analysed discriminately. Therefore, data from $N=617$ pupils of 28 different classes (third and fourth grade) were examined. As a result of multilevel analyses, there is a significant negative relationship between social insecurity and social integration in the dimensions relations, self-perception and acceptance by classmates. The results for the dimensions of social integration are discussed. Furthermore, implications for research and practice are derived.

Keywords: Social insecurity, social integration, primary school

Psychische Störungen gehören zu den häufigsten Erkrankungen im Kindes- und Jugendalter (Hölling, Schlack, Petermann, Ravens-Sieberer \& Mauz, 2014). Es ist anzunehmen, dass ein Großteil psychischer Störungen im Erwachsenenalter seinen Ursprung im Kinder- und Jugendalter hat. Diesem Lebensabschnitt ist somit eine sehr große Bedeutung für die Prävention und Intervention psychischer Störungen einzuräumen (Ihle \& Esser, 2007).

Internalisierende Störungen bzw. Verhaltensauffälligkeiten werden häufig als stille psychische Störungen beschrieben (Schneider \& In-Albon, 2010). Auffälligkeiten im internalisierenden Verhalten bleiben vom kindlichen Umfeld häufig unentdeckt, sodass keine ausreichenden Maßnahmen zur Prävention oder Intervention durchgeführt werden können (Weitkamp, Romer, Rosenthal, WiegandGrefe \& Daniels, 2010). Es ist anzunehmen, dass viele Schülerinnen und Schüler niederschwellige internalisierende Verhaltensauffälligkeiten aufweisen, die sich in kein diagnostisches Klassifikationsschema einordnen lassen (Petermann, 2005). In diesem Kontext ist die soziale Unsicherheit zu nennen, die im Fokus des vorliegenden Beitrags steht.
Während sich in Studien Hinweise auf einen negativen $\mathrm{Zu}$ sammenhang zwischen sozialen Ängsten und der sozialen Integration betroffener Kinder finden (z. B. La Greca \& Stone, 1993), ist bis dato weitestgehend unklar, wie die soziale Unsicherheit mit der sozialen Integration der betroffenen Kinder innerhalb ihrer Schulklasse zusammenhängt. Um sich dieser Frage anzunähern, fokussiert der vorliegende Beitrag die soziale Integration von sozial unsicheren Kindern.

\section{Theoretischer und empirischer Hintergrund}

\section{Soziale Unsicherheit}

Soziale Unsicherheit beschreibt das subjektive Unwohlsein in sozialen Situationen und die Sorge vor einer negativen Bewertung durch andere. Sofern es möglich ist, wer- 
den soziale Situationen vermieden oder auf ein Minimum beschränkt (Ahrens-Eipper, Leplow \& Nelius, 2010; La Greca \& Stone, 1993). Soziale Unsicherheit wird weder in der ICD-10 (World Health Organization, 2016) noch im DSM-V (American Psychiatric Association, 2013) als eigenes Störungsbild aufgeführt. Es sind jedoch Parallelen zu der Störung mit sozialer Ängstlichkeit oder der Sozialen Phobie zu erkennen, weswegen soziale Unsicherheit von einigen Autorinnen und Autoren als subklinische Form ebendieser Störungsbilder erachtet wird (Feiereis, Ahrens-Eipper \& Lässig, 2009). Ahrens-Eipper et al. (2010) erachten soziale Unsicherheit in der Kindheit als einen Risikofaktor für die Entwicklung psychischer Störungen (z. B. Angststörungen oder Depression) während der Adoleszenz oder im Erwachsenenalter.

Anders als bei einer Störung mit sozialer Ängstlichkeit kann soziale Unsicherheit auch nach dem sechsten Lebensjahr erstmalig auftreten, unter anderem, wenn im schulischen Kontext vermehrt soziale Situationen (Gruppenarbeiten o.ä.) stattfinden. Häufig auftretende Merkmale sind z.B. eine vermehrte Zurückhaltung und/oder geringe Beteiligung in Interaktionen (Ahrens-Eipper, 2002; Petermann \& Petermann, 2015). Einige Kinder mit sozialer Unsicherheit weisen hingegen keine sichtbare Beeinträchtigung in ihrem Alltag auf (Ahrens-Eipper et al., 2010; Petermann \& Petermann, 2015).

\section{Soziale Integration}

Der Begriff der sozialen Integration wird in der inklusionspädagogischen bzw. sonderpädagogischen Forschung sehr heterogen verwendet und operationalisiert (Koster et al., 2009; Kulawiak, Urton, Krull, Hennemann \& Wilbert, 2020).
Die Begriffe soziale Inklusion, soziale Partizipation und soziale Integration werden häufig synonym verwendet und beziehen sich dabei auf die soziale Dimension von Inklusion (Koster et al., 2009). In dem folgenden Beitrag wird, in Anlehnung an Marten, Voß und Blumenthal (2016), der Begriff soziale Integration genutzt, um die soziale Einbindung sowie die soziale Teilhabe eines Kindes in seiner Schulklasse zu beschreiben. Nach Koster et al. (2009) handelt es sich bei sozialer Integration um ein mehrdimensionales Konstrukt, welches sowohl die eigene Wahrnehmung des Kindes als auch die Wahrnehmung der anderen Kinder der Klasse einschließt. Nach Koster et al. (2009) umfasst das mehrdimensionale Konstrukt sozialer Integration die vier Ebenen Beziehungen, Kontakte, eigene Wahrnehmung sowie Akzeptanz. Weiter führen Koster et al. (2009) unterschiedliche Maße auf, anhand dessen die einzelnen Dimensionen sozialer Integration erhoben werden können (z.B. kann die Ebene eigene Wahrnehmung durch das Gefühl des Angenommenseins oder das soziale Selbstkonzept erhoben werden). Grütter, Meyer und Glenz (2015) weisen darauf hin, dass die Ergebnisse bezüglich der sozialen Integration je nach verwendetem Maß und somit auch Dimension variieren. Davon ausgehend erscheint es ratsam, die soziale Integration stets in ihrer Mehrdimensionalität zu berücksichtigen und alle vier Dimensionen zu erheben.

Eine mehrdimensionale Betrachtung sozialer Integration findet in jüngeren Studien zunehmend Beachtung (z.B. Henke et al., 2017). Auch Kulawiak und Wilbert (2015) verdeutlichen die Notwendigkeit einer differenzierten Betrachtungsweise sozialer Integration und führen das Modell nach Koster et al. (2009) als eine Möglichkeit auf.

Tabelle 1. Kurzbeschreibung der dargestellten Studien

\begin{tabular}{|c|c|c|}
\hline Studie & Stichprobe & Identifikation sozialer Angst \\
\hline Baker, Hudson \& Taylor (2014) & $N=96$, zwischen 7 und 12 Jahren alt, keine weiteren Angaben & Fragebogen, Interview \\
\hline Bartmanns et al. (2019) & $N=586, M_{\text {Atter }}=10.00, S D_{\text {Alter }}=1.20$, keine Angabe über die Jahrgangsstufe & Fragebogen \\
\hline Erath, Flanagan \& Bierman (2007) & $N=397$, keine Angabe über das Alter, Jahrgangsstufe 6 und 7 & Fragebogen \\
\hline La Greca \& Lopez (1998) & $N=250, M_{\text {Atter }}=17.04, S D_{\text {Alter }}=0.91$, Jahrgangsstufen 10 bis 12 & Fragebogen \\
\hline La Greca \& Stone (1993) & $N=587, M_{\text {Alter }}=10.99, S D_{\text {Alter }}=0.97$, Jahrgangsstufen 4 bis 6 & Fragebogen \\
\hline Rubin \& Mills (1988) & $\begin{array}{l}N=88, M_{\text {Alter }}=7.50, S D_{\text {Alter }}=0.40, \text { Jahrgangsstufe } 2, \text { es handelt sich } \\
\text { um eine längsschnittliche Studie bis zur Jahrgangsstufe } 5\end{array}$ & $\begin{array}{l}\text { Peer-Nominierungsverfahren, } \\
\text { Beobachtung }\end{array}$ \\
\hline $\begin{array}{l}\text { Rubin, Wojslawowicz, Rose- } \\
\text { Krasnor, Booth-LaForce \& } \\
\text { Burgess (2006) }\end{array}$ & $N=827, M_{\text {Alter }}=10.33, S D_{\text {Alter }}=0.52$, Jahrgangsstufe 5 & Peer-Nominierungsverfahren \\
\hline Rubin, Chen \& Hymel (1993) & $N=224, M_{\text {Atter }}=10.60$, keine Angabe über SD, Jahrgangsstufe 5 & Peer-Nominierungsverfahren \\
\hline $\begin{array}{l}\text { Strauss, Lahey, Frick, Frame \& } \\
\text { Hynd (1988) }\end{array}$ & $N=87$, zwischen 6 und 13 Jahren alt, keine weiteren Angaben & Diagnose nach DSM-III \\
\hline Verduin \& Kendall (2008) & $N=80$, zwischen 9 und 13 Jahren alt, keine weiteren Angaben & Diagnostisches Interview \\
\hline
\end{tabular}




\section{Soziale Unsicherheit und soziale Integration}

Für Kinder mit sozialer Unsicherheit liegen konkret noch keine Befunde hinsichtlich ihrer sozialen Integration vor. Jedoch finden sich mehrere Studien zur sozialen Integration von Kindern mit sozialen Ängsten. Diese werden im Folgenden entlang der vier Dimensionen sozialer Integration nach Koster et al. (2009) dargestellt. Informationen über die Stichprobe und die Identifikation sozialer Ängste in den einzelnen Studien sind in Tabelle 1 aufgeführt.

Beziehungen. Rubin, Wojslawowicz, Rose-Krasnor, BoothLaForce und Burgess (2006) untersuchten die Anzahl reziproker soziometrischer Wahlen als Maß der Beziehung. In ihrer Studie finden sich keine Unterschiede zwischen Kindern mit und ohne soziale(n) Ängste(n).

Kontakte. Interpretiert man abgegebene soziometrische Wahlen als einen Indikator für Kontaktinitiierung (Kulawiak \& Wilbert, 2015), kann für diese Dimension die Studie von La Greca und Lopez (1998) herangezogen werden. La Greca und Lopez (1998) berichten einen negativen Zusammenhang zwischen sozialen Ängsten und abgegebenen soziometrischen Wahlen.

Akzeptanz. Die Dimension der (sozialen) Akzeptanz wird häufig durch erhaltene soziometrische Wahlen operationalisiert (z. B. Marten et al., 2016). Experimentalstudien (Baker, Hudson \& Taylor, 2014; Verduin \& Kendall, 2008) zeigen, dass Kinder mit sozialen Ängsten weniger Wahlen erhalten. Feldstudien (La Greca \& Stone, 1993; Rubin \& Mills, 1988; Erath, Flanagan \& Bierman, 2007) bestätigen dieses Ergebnis. Dieser Befund findet sich ebenfalls bei Strauss, Lahey, Frick, Frame und Hynd (1988). Baartmans et al. (2019) konnten hingegen keinen Zusammenhang zwischen sozialen Ängsten und soziometrischen Wahlen nachweisen.

Eigene Wahrnehmung. Baartmans et al. (2019), La Greca und Lopez (1998) sowie La Greca und Stone (1993) berichten eine geringere selbstwahrgenommene soziale Integration von Kindern mit sozialen Ängsten. Dieser Befund findet sich auch in der Studie von Rubin, Chen und Hymel (1993). Des Weiteren berichten Rubin et al. (1993) sowie Rubin und Mills (1988) eine geringere Einschätzung der eigenen Sozialkompetenz sowie ein erhöhtes Gefühl der Einsamkeit von Kindern mit sozialen Ängsten. Dies deckt sich mit den Ergebnissen des Reviews von Kingery, Erdley, Marshall, Whitaker und Reuter (2010), wonach Kinder mit sozialen Ängsten ihre eigene Teilnahme an sozialen Situationen als nicht erfolgreich einschätzen. Die Ergebnisse der Studie von Erath et al. (2007) zeigen darüber hinaus einen Zusammenhang zwischen sozialen Ängsten und Viktimisierungserfahrungen in Bullyingprozessen.

Fazit. Trotz der unterschiedlichen Operationalisierungen weisen die dargestellten Befunde darauf hin, dass Schülerinnen und Schüler mit sozialen Ängsten ein erhöhtes Risiko einer unzureichenden sozialen Integration auf- weisen. Es ist anzunehmen, dass dieses Ergebnis auch auf Kinder mit sozialer Unsicherheit übertragen werden kann. Diese Annahme wird durch Kingery et al. (2010) gestützt, die in ihrem Review den Zusammenhang zwischen sozialen Beziehungen und Symptomen sozialer Angst bei Kindern ohne klinische Diagnose untersuchten.

Kritisch ist jedoch anzumerken, dass keine der hier dargestellten Studien die mehrdimensionale Struktur des Konzepts der sozialen Integration, wie z. B. bei Koster et al. (2009) beschrieben, in vollem Maße berücksichtigt bzw. eine konzeptuelle Verortung der untersuchten Teilaspekte vornimmt. Um ein möglichst differenziertes Bild über den Zusammenhang zwischen sozialer Integration und sozialer Unsicherheit zu erhalten, wird in der vorliegenden Arbeit die mehrdimensionale Betrachtung des Konstrukts sozialer Integration aufgegriffen und die von Koster et al. (2009) beschriebenen Dimensionen (Beziehung, Kontakt, eigene Wahrnehmung und Akzeptanz) berücksichtigt.

\section{Ziele und Fragestellung}

In der vorliegenden Studie soll der Zusammenhang zwischen sozialer Integration und sozialer Unsicherheit entlang der vier zentralen Dimensionen der sozialen Integration von Koster et al. (2009) untersucht werden. Konkret ergeben sich folgende Fragen und Hypothesen.

\section{Fragestellung 1 (reziproke Wahlen als Maß der Beziehungen)}

Gibt es einen Zusammenhang zwischen der Anzahl reziproker Beziehungen und sozialer Unsicherheit?

Da Schülerinnen und Schüler mit sozialer Unsicherheit ein vermehrtes Rückzugsverhalten zeigen und soziale Interaktionssituationen möglichst meiden (Ahrens-Eipper et al., 2010; La Greca \& Stone, 1993), ist davon auszugehen, dass sie nur wenig reziproke Beziehungen innerhalb ihrer Klasse pflegen. Es wird die Hypothese aufgestellt, dass ein negativer Zusammenhang zwischen sozialer Unsicherheit und den reziproken Beziehungen besteht (H1).

\section{Fragestellung 2 (abgegebene Wahlen als Maß der Kontakte)}

Besteht ein Zusammenhang zwischen dem Wunsch nach Kontakten und sozialer Unsicherheit?

Bei der Initiierung von Sozialkontakten mit ihren Peers verhalten sich Kinder mit sozialer Unsicherheit häufig passiv (Asendorpf, 1993; Petermann \& Petermann, 2015). 
Oftmals beteiligen sich ebendiese Kinder nicht an sozialen Interaktionen (z.B. Spielen auf dem Schulhof), sondern beobachten lediglich das soziale Miteinander (AhrensEipper et al., 2010; La Greca \& Lopez, 1988). Hieraus wird die Hypothese abgeleitet, dass ein negativer Zusammenhang zwischen sozialer Unsicherheit und den Kontaktinitiierungen besteht (H2).

\section{Fragestellung 3 (erhaltene Wahlen als Maß der Akzeptanz)}

Besteht ein Zusammenhang zwischen sozialer Akzeptanz und sozialer Unsicherheit?

Der Definition folgend vermeiden sozial unsichere Kinder Interaktionen mit anderen, sofern dies möglich ist (Ahrens-Eipper et al., 2010; La Greca \& Stone, 1993). Es ist anzunehmen, dass sich dies negativ auf die Akzeptanz ebendieser Kinder auswirkt. Für Kinder mit sozialen Ängsten konnte bereits mehrfach eine geringere soziale Akzeptanz nachgewiesen werden (z. B. Erath et al., 2007; La Greca \& Stone, 1993; Rubin \& Mills, 1988). Es wird daher die Hypothese aufgestellt, dass ein negativer Zusammenhang zwischen sozialer Unsicherheit und der Akzeptanz durch die Mitschülerinnen und Mitschüler besteht (H3).

\section{Fragestellung 4 (subjektive soziale Inte- gration als Maß der eigenen Wahrnehmung)}

Besteht ein Zusammenhang zwischen der selbstwahrgenommenen sozialen Integration und sozialer Unsicherheit?

Aus theoretischer und empirischer Perspektive finden sich Hinweise, dass Kinder mit sozialen Ängsten ihre eigene Teilnahme an sozialen Situationen als nicht erfolgreich einschätzen (Kingery et al., 2010). Es ist anzunehmen, dass sich dies auch auf sozial unsichere Kinder übertragen lässt. Neben der negativen Einschätzung der eigenen Teilnahme an sozialen Situationen wird vermutlich auch die subjektive soziale Integration negativ eingeschätzt. Daraus ergibt sich die Hypothese, dass ein negativer Zusammenhang zwischen sozialer Unsicherheit und der selbstwahrgenommenen sozialen Integration besteht $(\mathrm{H} 4)$.

\section{Methode}

\section{Stichprobe}

Insgesamt nahmen 790 Schülerinnen und Schüler aus 33 Schulklassen (dritte und vierte Jahrgangsstufe) aus
Nordrhein-Westfalen an einer Paper-Pencil-Befragung teil. Im Vorfeld an der Befragung wurden Informationsschreiben an die Klassenleitung und die Erziehungsberechtigten verschickt. Eine Teilnahme an der Befragung setzte das Einverständnis der Erziehungsberechtigten voraus. Der Ablauf und Inhalt der Befragung wurde im Vorfeld mit den Schülerinnen und Schülern mündlich besprochen. Es wurden nur Schulklassen mit in die Analysen aufgenommen, in welchen mindestens $75 \%$ der Schülerinnen und Schüler an der soziometrischen Befragung teilnahmen. Daher wurden insgesamt fünf Klassen mit 119 Schülerinnen und Schülern ausgeschlossen. Aufgrund unvollständiger Daten (mehr als ein fehlendes Item in dem Instrument zur Erfassung sozialer Unsicherheit) wurden weitere 54 Kinder ausgeschlossen.

Hieraus resultiert eine Stichprobe von $N=617$ Kindern ( $48 \%$ weiblich) aus 28 Schulklassen (acht Schulen) mit einer durchschnittlichen Klassengröße von $M=24$ $(S D=2.53)$. Aus datenschutzrechtlichen Gründen wurde das Alter nicht erfragt. Aufgrund der Jahrgangsstufen ist anzunehmen, dass die Kinder 8 bis 10 Jahre alt waren.

\section{Durchführung}

Bei der Befragung handelte es sich um eine papierbasierte Querschnittserhebung im Rahmen einer größeren Studie. Die Befragungen wurden durch geschulte Testleiterinnen im Klassenkontext durchgeführt und dauerten in etwa 90 Minuten. Um Leseverständnisproblemen vorzubeugen, wurden sämtliche Fragen vorgelesen. Die Befragungen fanden im Frühjahr 2018 innerhalb weniger Wochen statt.

\section{Instrumente}

In Anlehnung an Koster et al. (2009) wurde die soziale Integration von Schülerinnen und Schülern mit sozialer Unsicherheit anhand der vier Dimensionen Beziehungen, Kontakte, eigene Wahrnehmung und Akzeptanz untersucht.

Für die Erfassung von Beziehungen, Kontakten und Akzeptanz wurde ein soziometrisches Nominationsverfahren eingesetzt (Moreno, 1974). Die Kinder beantworteten für jedes Kind aus der Klasse die Frage, wie gerne sie neben diesem sitzen würden. Hierbei konnte zwischen den Antwortmöglichkeiten nicht gerne, es ist mir egal und sehr gerne (Wahlen) gewählt werden. Die Anzahl möglicher Wahlen $(n-1)$ bezieht sich nicht auf die tatsächliche Klassengröße, sondern auf die Anzahl der vorhandenen Soziometriebögen. Somit wurden die Berechnungen nur anhand der Kinder durchgeführt, die an der Querschnittserhebung teilgenommen hatten. 
Beziehungen. Die Beziehungen eines Kindes wurden anhand reziproker Wahlen operationalisiert. Unter reziproken Wahlen werden nach Kulawiak und Wilbert (2015) alle Wahlen verstanden, in denen sich zwei Schulkinder gegenseitig wählen. In die Datenanalyse ging dabei der Anteil der reziproken Wahlen an den in einer Klasse möglichen reziproken Wahlen ein. Hierfür wurde die Anzahl reziproker Wahlen durch die Anzahl möglicher reziproker Wahlen $(n-1)$ innerhalb einer Klasse dividiert.

Kontakte. In Anlehnung an Kulawiak und Wilbert (2015) werden die Kontaktwünsche eines Kindes als wichtiger Indikator für soziale Interaktionen und Kontakte betrachtet. Potentielle Kontakte wurden dieser Einschätzung folgend anhand abgegebener Wahlen eines Kindes operationalisiert. Analog zu der Berechnung der Beziehungen wurde auch der Anteil der Kontaktwünsche an den in einer Klasse möglichen Kontaktwünschen ermittelt, was der Anzahl abgegebener Wahlen dividiert durch die Anzahl möglicher Wahlen $(n-1)$ entspricht.

Akzeptanz. Die Akzeptanz wird im Sinne der erhaltenen Wahlen eines Kindes betrachtet. Hierzu wurden diese durch die Anzahl möglicher Wahlen $(n-1)$ innerhalb einer Klasse dividiert.

Eigene Wahrnehmung. Zur Überprüfung der selbstwahrgenommenen sozialen Integration wurde die Skala Soziale Integration (SI) des Fragebogens zur Erfassung emotionaler und sozialer Schulerfahrungen von Grundschulkindern (FEESS 3-4) von Rauer und Schuck (2003) eingesetzt, die elf Items auf einer vierstufigen Likertskala von stimmt gar nicht (0) bis stimmt genau (3) beinhaltet (z. B. „Meine Mitschüler sind nett zu mir."). Die interne Konsistenz beträgt $\alpha=.79$ für das dritte und $\alpha=.84$ für das vierte Schuljahr (Rauer \& Schuck, 2003) und im vorliegenden Datensatz $\alpha=.83$. Es liegen getrennte Normwerte für das dritte und das vierte Schuljahr vor. Eine geschlechtsspezifische Norm für den FEESS 3-4 liegt hingegen nicht vor.

Soziale Unsicherheit. Um die soziale Unsicherheit von Kindern zu erheben, wurde die Social Anxiety Scale for Children (SASC-R-D; Melfsen, 1998; Melfsen \& Florin, 1997) eingesetzt. Die SASC-R-D ist die deutschsprachige Übersetzung des gleichnamigen englischen Instruments (La Greca \& Stone, 1993). Der subklinische Selbstbeurteilungsbogen erfragt anhand von 18 Items die Auftretenshäufigkeit von Verhaltensweisen in sozialen Situationen (z.B. „Ich fühle mich unsicher bei Kindern, die ich nicht kenne.") auf einer fünfstufigen Likertskala $(O=n i e$, $4=$ immer) und richtet sich an Personen zwischen 8 und 16 Jahren. Das Verfahren kann als reliabel (Melfsen, 1998) und valide (Melfsen \& Walitza, 2010; Nicolay, Weber \& Huber, angenommen) beschrieben werden. Während die Autorinnen ursprünglich eine zweifaktorielle Struktur annahmen (Melfsen \& Florin, 1997), deuten neuere Untersuchungen eher auf eine bifaktorielle Struktur mit einem Globalfaktor hin (Nicolay, Weber \& Huber, angenommen). Entsprechend wurden für die vorliegende Untersuchung die 18 Items zu einem Gesamtwert verrechnet. Die interne Konsistenz im vorliegenden Datensatz beträgt $\alpha=.88$.

\section{Datenaufbereitung und Datenanalyse}

Zunächst fand eine Aufbereitung der Rohdaten statt. Für einzelne fehlende Werte in der SASC-R-D und im FEESS 3-4 wurde ein imputationsbasiertes Verfahren gewählt (Bortz \& Döring, 2016) und der fehlende Wert wurde durch den Mittelwert ersetzt. Sofern mehr als ein Wert in einer der Skalen fehlte, wurde der Fall aus der Stichprobe ausgeschlossen. Fehlende Daten in der Soziometrie wurden nicht ersetzt, sondern führten zu einem Ausschluss des jeweiligen Falls. Dadurch ergeben sich unterschiedliche Größen für die Gesamtstichprobe $N=617$ (beinhaltet alle gültigen SASC-R-D Datensätze) und die Teilstichprobe $n_{\text {FEESS }}=608$ sowie $n_{\text {Soziometrie }}=609$.

Die Überprüfung der Fragestellungen erfolgte jeweils mit einem Random-Intercept-Mehrebenenregressionsmodell. Dadurch konnte die genestete Struktur der Daten berücksichtigt werden, die sich durch die Klassenzugehörigkeit der Schülerinnen und Schüler ergibt. In den insgesamt vier Regressionsanalysen bildete der jeweilige Teilaspekt der sozialen Integration die abhängige Variable. Die an der jeweiligen Anzahl der Schülerinnen und Schüler pro Klasse relativierten Soziometriemaße sind als Prozentwerte gut interpretierbar. Ausgehend von der Präferenz für gleichgeschlechtliche Freundschaften (McPherson, Smith-Lovin \& M Cook, 2001) erfolgte die Auswertung dabei für Jungen und Mädchen innerhalb einer Klasse getrennt. Hierzu wurde die jeweilige soziometrische Variable von Kindern des gleichen Geschlechts durch die Anzahl möglicher Werte für diese soziometrische Variable $(n-1)$ innerhalb einer Klasse dividiert. Aufgrund unterschiedlicher Normwerte für das dritte und vierte Schuljahr im FEESS 3-4 (Rauer \& Schuck, 2003) wurden die entsprechenden T-Werte der Skala Soziale Integration als abhängige Variable einbezogen. Die Rohwerte zur sozialen Unsicherheit wurden als unabhängige Variable z-transformiert in das Modell aufgenommen. Zusätzlich wurde das Geschlecht dummycodiert (Referenzkategorie: männlich) als Kontrollvariable in alle Modelle aufgenommen.

Die Datenanalyse erfolgte mit R 3.6.1 (R Core Team, 2019) unter Hinzunahme der R-Pakete lme 4 1.1-23 (Bates, Mächler, Bolker \& Walker, 2015), lmerTest 3.1-2 (Kuznetsova, Brockhoff \& Christensen, 2017) und MuMIn 1.43.17 (Bartoń, 2020). 


\section{Ergebnisse}

Im Folgenden werden die Berechnungen zur Überprüfung der Zusammenhänge zwischen sozialer Unsicherheit und den einzelnen Dimensionen sozialer Integration aufgeführt. In Tabelle 2 werden zunächst die deskriptiven Werte aller einbezogenen Variablen dargestellt.

Tabelle 2 zeigt, dass die wahrgenommene soziale Integration mit einem durchschnittlichen T-Wert von 48 nur knapp unter dem Mittelwert der Eichstichprobe von 50 liegt. Abgegebene und erhaltene Wahlen sind über die Gesamtstichprobe identisch und liegen jeweils bei $40 \%$. Das bedeutet, dass die Kinder einer Klasse etwas weniger als die Hälfte ihrer Klassenkameradinnen oder -kameraden gerne als Sitznachbarin oder -nachbarn wählen würden. Dies wird aber nur in rund $23 \%$ der Fälle (reziproke Wahlen) erwidert.

\section{Fragestellung 1 (Beziehungen)}

In Fragestellung 1 wird geprüft, ob ein Zusammenhang zwischen sozialer Unsicherheit und dem Anteil reziproker Wahlen besteht. Das Nullmodell zeigt eine Intraklassenkorrelation (ICC) von .05. Somit können 5\% der Varianz reziproker Wahlen zwischen den Schülerinnen und Schülern (Level 1) durch Unterschiede zwischen den Schulklassen (Level 2) erklärt werden. Der Random-Intercept von $\beta=21.81$ besagt, dass durchschnittlich sozial unsichere Jungen (Referenzkategorie) ca. 22\% reziproke Wahlen erhalten (Tabelle 3). Wenn die soziale Unsicherheit um eine Standardabweichung ansteigt, verringert sich der Anteil reziproker Wahlen um ca. 2\% ( $\beta=-2.19)$. Dieser Zusammenhang ist signifikant. Es finden sich keine Geschlechtseffekte für den Anteil reziproker Wahlen.

\section{Fragestellung 2 (Kontakte)}

Es wird angenommen, dass ein negativer Zusammenhang zwischen abgegebenen Wahlen (als Maß der Kontakte) und der sozialen Unsicherheit besteht. Das Nullmodell zeigt eine ICC von .02. Im Random-Intercept-Modell zeigt sich, dass durchschnittlich sozial unsichere Jungen ca. 38\% ( $\beta=37.98$ ) Wahlen vergeben (Tabelle 3). Durch den Anstieg sozialer Unsicherheit um eine Standardabweichung verringert sich der Anteil abgegebener Wahlen um ca. 2\% $(\beta=-1.78)$. Dieser Zusammenhang ist nicht signifikant. Mädchen der vorliegenden Stichprobe vergeben ca. 5\% $(\beta=4.97)$ mehr Wahlen als Jungen. Dieser Effekt ist signifikant.

\section{Fragestellung 3 (erhaltene Wahlen als Facette der Akzeptanz)}

In Fragestellung 3 wird untersucht, ob ein Zusammenhang zwischen sozialer Unsicherheit und den erhaltenen Wahlen besteht. Das Nullmodell zeigt eine ICC von .03. Im Random-Intercept-Modell zeigt sich, dass durchschnittlich sozial unsichere Jungen ca. 37\% Wahlen $(\beta=37.31)$ erhalten. Wenn die soziale Unsicherheit um eine Standardabweichung steigt, verringert sich der Anteil erhaltener Wahlen um ca. $3 \%(\beta=-3.23)$. Dieser Zusammenhang ist signifikant. Mädchen erhalten ca. $5 \%(\beta=5.39)$ mehr Wahlen als Jungen (Tabelle 3). Dieses Ergebnis ist ebenfalls signifikant.

\section{Fragestellung 4 (eigene Wahrnehmung)}

Es wird geprüft, ob ein Zusammenhang zwischen sozialer Unsicherheit und der selbstwahrgenommenen sozialen Integration besteht. Das Nullmodell zeigt eine ICC von .05. Der Intercept von $\beta=47.26$ spiegelt die wahrgenommene soziale Integration von durchschnittlich sozial unsicheren Jungen (als T-Wert) wider (Random-InterceptModell in Tabelle 3). Wenn die soziale Unsicherheit um eine Standardabweichung steigt, verringert sich die selbstwahrgenommene soziale Integration um ca. drei T-Werte $(\beta=-2.69)$. Dieser Zusammenhang ist signifikant. Es zeigt sich zudem ein signifikanter Geschlechtseffekt hinsichtlich der wahrgenommenen sozialen Integration. Bei Mädchen ist diese um ca. zwei T-Werte höher als bei Jungen (Tabelle 3).

Tabelle 2. Darstellung der deskriptiven Ergebnisse aller erhobenen Variablen

\begin{tabular}{|c|c|c|c|c|}
\hline & $N$ & $M(S D)$ & Min & $\operatorname{Max}$ \\
\hline \multicolumn{5}{|l|}{ Soziale Integration } \\
\hline Wahrgenommene soziale Integration (T-Wert) & 608 & $48(10)$ & 20 & 69 \\
\hline Reziproke Wahlen & 609 & $23 \%(17 \%)$ & $0 \%$ & $82 \%$ \\
\hline Abgegebene Wahlen & 609 & $40 \%(23 \%)$ & $0 \%$ & $100 \%$ \\
\hline Erhaltene Wahlen & 609 & $40 \%(23 \%)$ & $0 \%$ & $100 \%$ \\
\hline Soziale Unsicherheit (Gesamtwert) & 617 & $22.03(13.21)$ & 0 & 62 \\
\hline
\end{tabular}


Tabelle 3. Ergebnisse der Random-Intercept-Modelle für die abhängigen Variablen (AV)

\begin{tabular}{|c|c|c|c|c|c|c|c|c|c|c|c|c|}
\hline \multirow[b]{2}{*}{ Prädiktoren } & \multicolumn{3}{|c|}{ Reziproke Wahlen } & \multicolumn{3}{|c|}{ Abgegebene Wahlen } & \multicolumn{3}{|c|}{ Erhaltene Wahlen } & \multicolumn{3}{|c|}{$\begin{array}{l}\text { Selbstwahrgenommene } \\
\text { soziale Integration }\end{array}$} \\
\hline & $\beta$ & $\mathrm{Cl}$ & $p$ & $\beta$ & $\mathrm{Cl}$ & $p$ & $\beta$ & $\mathrm{Cl}$ & $p$ & $\beta$ & $\mathrm{Cl}$ & $p$ \\
\hline (Intercept) & 21.81 & $19.41-24.21$ & $<.001 \star$ & 37.98 & $34.80-41.16$ & $<.001 \star$ & 37.31 & $34.37-40.26$ & $<.001 \star$ & 47.26 & $45.83-48.69$ & $<.001^{\star}$ \\
\hline $\begin{array}{l}\text { Soziale } \\
\text { Unsicherheit } \\
\text { (z-trans- } \\
\text { formiert) }\end{array}$ & -2.19 & $-3.55--0.83$ & $.002 *$ & -1.78 & $-3.66-0.10$ & .064 & -3.23 & $-5.09--1.36$ & $.001 *$ & -2.69 & $-3.49--1.90$ & $<.001 *$ \\
\hline $\begin{array}{l}\text { Geschlecht } \\
\text { (w) }\end{array}$ & 2.64 & $-0.05-5.33$ & .054 & 4.97 & $1.26-8.69$ & $.009 *$ & 5.39 & $1.70-9.08$ & $.004^{*}$ & 2.01 & $0.44-3.59$ & $.012^{\star}$ \\
\hline \multicolumn{13}{|l|}{ Zufallseffekte } \\
\hline$\sigma^{2}$ & \multicolumn{3}{|c|}{260.76} & \multicolumn{3}{|c|}{498.63} & \multicolumn{3}{|c|}{495.89} & \multicolumn{3}{|c|}{89.43} \\
\hline$\tau_{00}$ & \multicolumn{3}{|c|}{17.34} & \multicolumn{3}{|c|}{26.77} & \multicolumn{3}{|c|}{16.96} & \multicolumn{3}{|c|}{6.47} \\
\hline ICC & \multicolumn{3}{|c|}{0.06} & \multicolumn{3}{|c|}{0.05} & \multicolumn{3}{|c|}{0.03} & \multicolumn{3}{|c|}{0.07} \\
\hline$N_{\text {Klassen }}$ & \multicolumn{3}{|c|}{28} & \multicolumn{3}{|c|}{28} & \multicolumn{3}{|c|}{28} & \multicolumn{3}{|c|}{28} \\
\hline$n_{\text {sus }}$ & \multicolumn{3}{|c|}{609} & \multicolumn{3}{|c|}{609} & \multicolumn{3}{|c|}{609} & \multicolumn{3}{|c|}{608} \\
\hline $\mathrm{R}^{2} \mathrm{~m} / \mathrm{R}^{2} \mathrm{C}$ & \multicolumn{3}{|c|}{$0.018 / 0.079$} & \multicolumn{3}{|c|}{$0.013 / 0.064$} & \multicolumn{3}{|c|}{$0.025 / 0.057$} & \multicolumn{3}{|c|}{$0.068 / 0.130$} \\
\hline $\mathrm{AIC} / \mathrm{BIC}$ & \multicolumn{3}{|c|}{$5143.06 / 5165.61$} & \multicolumn{3}{|c|}{$5532.67 / 5555.22$} & \multicolumn{3}{|c|}{$5522.53 / 5545.08$} & \multicolumn{3}{|c|}{$4488.54 / 4511.08$} \\
\hline
\end{tabular}

Anmerkungen: $w=$ weiblich; $*=p<.05$

\section{Diskussion}

Das Ziel des vorliegenden Beitrags bestand darin, Zusammenhänge zwischen sozialer Unsicherheit und sozialer Integration von Schülerinnen und Schülern der Primarstufe zu untersuchen. Hierzu wurden 617 Kinder aus insgesamt 28 Schulklassen der Jahrgangsstufen drei und vier in einer Querschnittsstudie befragt. Die Mehrdimensionalität sozialer Integration (Koster et al., 2009) wurde berücksichtigt und es wurden Random-Intercept-Mehrebenenregressionsmodelle für die vier Dimensionen Beziehungen, Kontakte, eigene Wahrnehmung und Akzeptanz berechnet.

\section{Zusammenfassung und Diskussion der Ergebnisse}

Die Ergebnisse der Mehrebenenregressionsanalyse zeigen, dass soziale Unsicherheit und der Anteil reziproker Wahlen (als Maß der Beziehung) signifikant negativ miteinander in Verbindung stehen. Hypothese 1 kann somit bestätigt werden.

Entgegen der formulierten Hypothese 2 findet sich in der vorliegenden Studie kein Zusammenhang zwischen der sozialen Unsicherheit und der Kontaktinitiierung (operationalisiert durch die Abgabe von Wahlen).

Die Ergebnisse der Fragestellung 3 zeigen einen signifikant negativen Zusammenhang zwischen sozialer Unsi- cherheit und der Akzeptanz durch die Mitschülerinnen und Mitschüler (operationalisiert durch erhaltene Wahlen). Somit kann Hypothese 3 bestätigt werden.

Der in dieser Studie angenommene negative Zusammenhang zwischen sozialer Unsicherheit und der subjektiv wahrgenommenen sozialen Integration findet sich in den Ergebnissen wieder, weshalb Hypothese 4 bestätigt werden kann.

Außer in der Dimension Beziehungen konnten in den drei verbleibenden Dimensionen Geschlechtseffekte nachgewiesen werden. Es zeigt sich, dass Mädchen der vorliegenden Stichprobe mehr Wahlen vergeben und erhalten als Jungen. Überraschend ist der Geschlechtseinfluss auf die eigene Wahrnehmung, da sich im Manual des FEESS 3-4 (Rauer \& Schuck, 2003) hierzu keine Hinweise finden. Die vorliegenden Ergebnisse decken sich jedoch mit denen von Krawinkel, Südkamp, Lange und Troster (2017), wonach Jungen ihre soziale Integration in der Schulklasse geringer einschätzen als Mädchen.

Trotz der mehrheitlich signifikanten negativen Zusammenhänge sollten die Ergebnisse der vorliegenden Studie insgesamt nicht überschätzt werden. Exemplarisch seien die Ergebnisse für die selbstwahrgenommene soziale Integration aufgeführt. Ein Anstieg der sozialen Unsicherheit um eine Standardabweichung verringert die selbstwahrgenommene soziale Integration lediglich um drei T-Werte. Analog zeigt sich, dass die berechneten Modelle insgesamt nur wenig Varianz der jeweiligen abhängigen Variablen erklären (.06 - .13). Eine Erklärung hierfür könnte sein, dass 
abseits von sozialer Unsicherheit und Geschlecht keine weiteren Prädiktoren einbezogen wurden.

\section{Methodische Einschränkungen und Ausblick}

Die vorgenommene Fokussierung auf die soziale Unsicherheit der Kinder ohne weiteren Einbezug möglicher Prädiktoren stellt eine Limitation der vorliegenden Studie dar. Einige empirische Befunde weisen darauf hin, dass neben schulkindbezogenen Variablen auch das Lehrkraftfeedback (Huber, Gebhardt \& Schwab, 2015; Huber, Gerullis, Gebhardt \& Schwab, 2018; Nicolay \& Huber, angenommen) oder Aspekte der Unterrichtsorganisation, wie z.B. der Einsatz kooperativer Lernformen (Weber \& Huber, 2020) die soziale Integration beeinflussen können. Ein Überblick findet sich bei Huber (2019). Da der Fokus der vorliegenden Studie auf der sozialen Unsicherheit der Schülerinnen und Schüler lag, wurden diese klassenspezifischen Variablen bewusst nicht berücksichtigt. In Folgestudien könnten jedoch auch Level 2-Prädiktoren in die Mehrebenenanalyse einbezogen werden.

Trotz der Berücksichtigung der Mehrdimensionalität sozialer Integration, wie von Koster et al. (2009) empfohlen, konnten in der vorliegenden Studie nur ausgewählte Aspekte der vier Dimensionen berücksichtigt werden. Es wäre wünschenswert, weitere Aspekte in Folgestudien zu untersuchen.

Für die Überprüfung der Dimensionen Beziehungen, Kontakte und Akzeptanz wurden soziometrischen Befragungen durchgeführt. Die soziometrische Befragung fand in der vorliegenden Studie anhand der Frage nach dem Sitznachbarn oder der Sitznachbarin statt. Diese ausgewählte Fragestellung ist diskutabel. Zwar konnten Krawinkel et al. (2017) eine hohe Interkorrelation unterschiedlicher soziometrischer Fragestellungen nachweisen, dennoch wäre es möglich, dass die Dimensionen Beziehungen und Kontakte durch die Frage nach dem Sitznachbarn oder der Sitznachbarin womöglich nicht vollständig abgebildet werden. Es gilt zu bedenken, dass es sich bei der Wahl einer Sitznachbarin oder eines Sitznachbarn um eine schulspezifische und nicht freiwillige soziale Situation handelt, die womöglich durch weitere Komponenten (z.B. Hilfsbereitschaft) beeinflusst wird. Da soziale Unsicherheit häufig in nicht freiwählbaren Situationen auftritt, in denen eine soziale Interaktion nicht vermieden werden kann, erschien die Auswahl ebendieser Frage für die vorliegende Studie passend. Um die Validität der soziometrischen Daten zu erhöhen, erscheint es jedoch sinnvoll, in Folgestudien weitere soziometrische Fragestellungen mit einzubeziehen, die sich auf frei wählbare soziale Situationen beziehen. Marten et al. (2016) ergänzten die Frage nach dem Sitznachbarn bzw. der Sitznachbarin z.B. durch die Frage nach Kindern, die man gerne zu seinem Geburtstag einladen würde.

Rubin et al. (2006) sowie Hoffmann, Wilbert, Lehofer und Schwab (2020) erhoben neben der Quantität reziproker Beziehungen auch deren Qualität. Anhand der Daten der vorliegenden Studie können lediglich Aussagen über die Quantität bestehender Beziehungen getroffen werden. In Folgestudien sollte neben dem quantitativen auch der qualitative Bereich von Beziehungen erhoben werden, um die Dimension Beziehung möglichst differenziert zu erfassen. Darüber hinaus bieten sich Netzwerkanalysen (Kulawiak, 2015) zur genaueren Analyse bestehender Beziehungen an.

Des Weiteren ist zu berücksichtigen, dass es sich in der vorliegenden Studie um eine Querschnittserhebung handelt. Um differenziertere Aussagen über die Beziehungen zwischen sozialer Unsicherheit und sozialer Integration treffen zu können, sollten Längsschnittstudien eingesetzt werden. Ladd und Troop-Gordon (2003) wiesen in einer solchen Studie nach, dass ängstliches Verhalten im Elementarbereich nicht zwangsweise einen Prädiktor für eine unzureichende soziale Integration im Primarbereich darstellt. Dies steht in einem Widerspruch zu den Ergebnissen von DeRosier, Kupersmidt und Patterson (1994), wonach ängstliches Verhalten das Risiko einer unzureichenden sozialen Integration begünstigt.

\section{Fazit}

Wie eingangs aufgeführt, stellt die soziale Unsicherheit von Kindern einen Risikofaktor für die Entwicklung psychischer Störungen dar (Ahrens-Eipper et al., 2010). Dieser Beitrag erweitert das Wissen in Bezug auf die soziale Unsicherheit im schulischen Kontext, indem erstmals auch Zusammenhänge mit den einzelnen Dimensionen sozialer Integration untersucht wurden. Die mehrheitlich negativen Zusammenhänge zeigen die Notwendigkeit auf, Schülerinnen und Schüler mit Merkmalen sozialer Unsicherheit auch in Bezug auf deren soziale Integration aufmerksam zu beobachten. Während soziale Unsicherheit in einem negativen Zusammenhang zu den Beziehungen und der Akzeptanz mit bzw. durch die Mitschülerinnen und Mitschüler sowie zur subjektiven sozialen Integration steht, findet sich in den Ergebnissen kein Zusammenhang zwischen sozialer Unsicherheit und dem Wunsch nach Kontakten zu Peers. Dies könnte ein Hinweis darauf sein, dass der Wunsch nach sozialer Interaktion ungeachtet der sozialen Unsicherheit besteht. Bisherige Befunde zur sozialen Unsicherheit, wonach betroffene Kinder in sozialen Interaktionen häufig zurückhaltend agieren (Ahrens-Eipper et al., 2010), deuten jedoch darauf hin, dass sozial unsichere Kinder diese Situationen womöglich nicht erfolg- 
reich bewerkstelligen können. Eine Erklärung könnte sein, dass Schülerinnen und Schüler mit sozialer Unsicherheit, ebenso wie Kinder mit sozialen Ängsten (Kingery et al., 2010), über eingeschränkte soziale Kompetenzen verfügen bzw. diese unterschätzen (Hymel, Bowker \& Woody, 1993; Rubin \& Mills, 1988; Rubin et al., 1993). Inwieweit soziale Unsicherheit aus unzureichenden sozialen Kompetenzen resultiert oder diese begünstigt, ist bis dato noch unklar und sollte in weiteren Studien untersucht werden. Für Kinder mit Merkmalen sozialer Unsicherheit erscheint es besonders wichtig, Interaktionssituationen im schulischen Kontext zu gestalten, in denen sich die Kinder sicher fühlen und die sie erfolgreich bewerkstelligen können. Dies könnte ein erster Impuls für die Förderung ebendieser Kinder im schulischen Kontext darstellen. In weiteren Studien sollte dies überprüft werden.

\section{Literatur}

Ahrens-Eipper, S. (2002). Soziale Unsicherheit im Kindesalter. Indikation und Effektivität eines verhaltenstherapeutischen Trainings (Dissertation an der Martin-Luther-Universität Halle-Wittenberg). Berlin: Dissertation.de.

Ahrens-Eipper, S., Leplow, B. \& Nelius, K. (2010). Mutig werden mit Til Tiger. Ein Trainingsprogramm für sozial unsichere Kinder (2. ,erweiterte Aufl.). Göttingen: Hogrefe.

American Psychiatric Association. (2013). Diagnostic and Statistical Manual of Mental Disorders. DSM-5. Washington, D. C.: American Psychiatric Publishing. https://doi.org/10.1176/appi.books.9780 890425596

Asendorpf, J. B. (1993). Abnormal shyness in children. Journal of Child Psychology and Psychiatry, 34, 1069 -1083. https://doi. org/10.1111/j.1469-7610.1993.tb01774.x

Baartmans, J. M. D., Rinck, M., Hudson, J. L., Lansu, T. A. M., van Niekerk, R. E., Bögels, S. M. et al. (2019). Are socially anxious children really less liked, or do they only think so? Cognitive Therapy and Research, 43,1043-1050. https://doi.org/10.1007/ s10608-019-10028-9

Baker, J. R., Hudson, J. L. \& Taylor, A. (2014). An investigation into the lower peer liking of anxious than nonanxious children. Journal of Anxiety Disorders, 28, 599-611. https://doi.org/10.1016/j. janxdis.2014.06.007

Bartoń, K. (2020). MuMIn: Multi-Model Inference. R package version 1.43.17. Verfügbar unter https://CRAN.R-project.org/ package $=$ MuMln

Bates, D., Mächler, M., Bolker, B. \& Walker, S. (2015). Fitting linear mixed-effects models using Ime4. Journal of Statistical Software, 67 (1), 1 - 48. https://doi.org/10.18637/jss.v067.i01

Bortz, J. \& Döring, N. (2016). Forschungsmethoden und Evaluation. Für Human- und Sozialwissenschaftler (vollständig überarbeitete, aktualisierte und erweiterte Aufl.). Heidelberg: Springer.

DeRosier, M. E., Kupersmidt, J. B. \& Patterson, C. J. (1994). Children's academic and behavioral adjustment as a function of the chronicity and proximity of peer rejection. Child Development, 65, 1799-1813. https://doi.org/10.2307/1131295

Erath, S. A., Flanagan, K. S. \& Bierman, K. L. (2007). Social anxiety and peer relations in early adolescence: behavioral and cognitive factors. Journal of Abnormal Child Psychology, 35, 405- 416. https://doi.org/10.1007/s10802-007-9099-2
Feiereis, E. U., Ahrens-Eipper, S. \& Lässig, W. (2009). Auswirkungen eines Klinikaufenthaltes auf die soziale Unsicherheit im Kindesalter. Kindheit und Entwicklung, 18, 13-20. https://doi. org/10.1026/0942-5403.18.1.13

Grütter, J., Meyer, B. \& Glenz, A. (2015). Sozialer Ausschluss in Integrationsklassen: Ansichtssache? Psychologie in Erziehung und Unterricht, 62, 65-82. https://doi.org/10.2378/peu2015.art05d Henke, T., Bosse, S., Lambrecht, J., Jäntsch, C., Jaeuthe, J. \& Spörer, N. (2017). Mittendrin oder nur dabei? Zum Zusammenhang zwischen sonderpädagogischem Förderbedarf und sozialer Partizipation von Grundschülerinnen und Grundschülern. Zeitschrift für Pädagogische Psychologie, 31, 111 -123. https:// doi.org/10.1024/1010-0652/a000196

Huber, C. (2019). Ein integriertes Rahmenmodell zur Förderung sozialer Integration im inklusiven Unterricht. Sozialpsychologische Grundlagen, empirische Befunde und schulpraktische Ableitungen. Vierteljahresschrift für Heilpädagogik und ihre Nachbargebiete, 88, 27 - 43. https://doi.org/10.2378/vhn2019. art06d

Huber, C., Gebhardt, M. \& Schwab, S. (2015). Lehrkraftfeedback oder Spaß beim Spiel? Eine Experimentalstudie zum Einfluss von Lehrkraftfeedback auf die soziale Akzeptanz bei Grundschulkindern. Psychologie in Erziehung und Unterricht, 61, 51 -64. https://doi.org/10.2378/peu2015.art04d

Huber, C., Gerullis, A., Gebhardt, M. \& Schwab, S. (2018). The impact of social referencing on social acceptance of children with disabilities and migrant background. An experimental study in primary school settings. European Journal of Special Needs Education, 1,1 - 17. https://doi.org/10.1080/08856257.2018.14 24778

Hölling, H., Schlack, R., Petermann, F., Ravens-Sieberer, U. \& Mauz, E. (2014). Psychische Auffälligkeiten und psychosoziale Beeinträchtigungen bei Kindern und Jugendlichen im Alter von 3 bis 17 Jahren in Deutschland - Prävalenz und zeitliche Trends zu 2 Erhebungszeitpunkten (2003-2006 und 2009-2012): Ergebnisse der KiGGS-Studie - Erste Folgebefragung (KiGGS Welle 1). Bundesgesundheitsblatt, Gesundheitsforschung, Gesundheitsschutz, 57, 807-819. https://doi.org/10.1007/s00 103-014-1979-0̈3

Hoffmann, L., Wilbert, J., Lehofer, M. \& Schwab, S. (2020). Are we good friends? - Friendship preferences and the quantity and quality of mutual friendships. European Journal of Special Needs Education. https://doi.org/10.1080/08856257.2020.1769980

Hymel, S., Bowker, A. \& Woody, E. (1993). Aggressive versus withdrawn unpopular children: Variations in peer and self-perceptions in multiple domains. Child Development, 64, 879-896. https://doi.org/10.2307/1131224

Ihle, W. \& Esser, G. (2007). Psychische Störungen im Kindes- und Jugendalter: Gesundheitliche Lage, gesundheitliche Versorgung und Empfehlungen. In Berufsverband deutscher Psychologinnen und Psychologen (Hrsg.), Bericht zur Kinder- und Jugendgesundheit in Deutschland (S.11 - 21). Berlin: Berufsverband deutscher Psychologinnen und Psychologen.

Kingery, J. N., Erdley, C. A., Marshall, K. C., Whitaker, K. G. \& Reuter, T. R. (2010). Peer experiences of anxious and socially withdrawn youth: An integrative review of the developmental and clinical literature. Clinical Child and Family Psychology Review, 13, 91 - 128. https://doi.org/10.1007/s10567-009-0063-2

Koster, M., Nakken, H., Pijl, S. J. \& van Houten, E. (2009). Being part of the peer group. A literature study focusing on the social dimension of inclusion in education. International Journal of Inclusive Education, 13,117-140. https://doi.org/10.1080/13603110701284680

Krawinkel, S., Südkamp, A., Lange, S. \& Troster, H. (2017). Soziale Partizipation in inklusiven Grundschulklassen: Bedeutung von Klassen- und Lehrkraftmerkmalen. Empirische Sonderpädagogik, 9, 277 - 295.Verfügbar unter urn:nbn:de:0111-pedocs-151721 
Kulawiak, P. R. (2015). Soziometrie und Netzwerkanalyse - Eine Methode zur Erfassung der Gruppendynamik in inklusiven Schulklassen. Potsdamer Zentrum für empirische Inklusionsforschung, 1, 1-9. Verfügbar unter https://www.uni-potsdam. de/fileadmin01/projects/inklusion/PDFs/ZEIF-Blog/Kulawiak _2015_Soziometrie_und_Netzwerkanalyse.pdf

Kulawiak, P. R., Urton, K., Krull, J., Hennemann, T. \& Wilbert, J. (2020). Internalizing behavior of sociometrically neglected students in inclusive primary classrooms - A methodological issue? Frontiers in Education, 5. https://doi.org/10.3389/feduc.2020.00032

Kulawiak, P. R. \& Wilbert, J. (2015). Methoden zur Analyse der sozialen Integration von Schulkindern mit sonderpädagogischem Förderbedarf im gemeinsamen Unterricht. Empirische Sonderpädagogik, 7, 241-257. Verfügbar unter urn:nbn:de:0111pedocs-113857

Kuznetsova, A., Brockhoff, P. B. \& Christensen, R. H. B. (2017). ImerTest Package: Tests in linear mixed effects models. Journal of Statistical Software, 82, 1 - 26. https://doi.org/10.18637/jss.v082.i13

La Greca, A. M. \& Lopez, N. (1998). Social anxiety among adolescents: Linkages with peer relations and friendships. Journal of Abnormal Child Psychology, 26, 83-94. https://doi.org/10. 1023/A:1022684520514

La Greca, A. M. \& Stone, W. L. (1993). Social Anxiety Scale for Children-Revised: Factor structure and concurrent validity. Journal of Clinical Child Psychology, 22,17 - 27. https://doi.org/10.1207/ s15374424jccp2201_2

Ladd, G. W. \& Troop-Gordon, W. (2003). The role of chronic peer difficulties in the development of children's psychological adjustment problems. Child Development, 74,1344-1367. https://doi. org/10.1111/1467-8624.00611

Marten, K., Voß, S. \& Blumenthal, Y. (2016). Zur Anwendung des Nominierungsverfahrens als Methode zur Erfassung der sozialen Akzeptanz. Empirische Pädagogik, 30, 8- 26.

McPherson, M., Smith-Lovin, L. \& M Cook, J. (2001). Birds of a Feather: Homophily in Social Networks. Annual Review of Sociology, 27, 415 - 444. https://doi.org/10.1146/annurev.soc.27.1.415

Melfsen, S. (1998). Die deutsche Fassung der Social Anxiety Scale for Children Revised (SASC-R-D): Psychometrische Eigenschaften und Normierung. Diagnostica, 44, $153-163$.

Melfsen, S., \& Florin, I. (1997). Ein Fragebogen zur Erfassung sozialer Angst bei Kindern (SASC-R-D). Kindheit und Entwicklung: Zeitschrift Für Klinische Kinderpsychologie, 6, 224-229.

Melfsen, S. \& Walitza, S. (2010). Skalen zur Erfassung von Angststörungen im Kindes- und Jugendalter. Klinische Diagnostik und Evaluation, 3, $142-163$

Moreno, J. L. (1974). Die Grundlagen der Soziometrie. Wege z. Neuordnung d. Gesellschaft (3. Aufl.). Opladen: Westdeutscher Verlag.

Nicolay, P. \& Huber, C. (angenommen). Wie Schulleistung und Lehrkraftfeedback die soziale Akzeptanz beeinflussen: Ergebnisse einer Experimentalstudie. Empirische Sonderpädagogik.

Nicolay, P., Weber, S., \& Huber, C. (angenommen). Überprüfung der Konstrukt-, Kriteriumsvalidität und Messinvarianz eines Instruments zur Messung von sozialer Unsicherheit. Diagnostica.

Petermann, F. (2005). Zur Epidemiologie psychischer Störungen im Kindes- und Jugendalter. Kindheit und Entwicklung, 14, 48-57. https://doi.org/10.1026/0942-5403.14.1.48

Petermann, U. \& Petermann, F. (2015). Training mit sozial unsicheren Kindern. Behandlung von sozialer Angst, Trennungsangst und generalisierter Angst (11., überarbeitete und erweiterte Aufl.). Weinheim und Basel: Beltz.

R Core Team. (2019). A language and environment for statistical computing. R Foundation for Statistical Computing [Computer software]. Vienna: Foundation for Statistical Computing. Available from https://www.R-project.org/
Rauer, W. \& Schuck, K. D. (2003). Fragebogen zur Erfassung emotionaler und sozialer Schulerfahrungen von Grundschulkindern dritter und vierter Klassen. Göttingen: Hogrefe.

Rubin, K. H., Chen, X. \& Hymel, S. (1993). Socioemotional characteristics of withdrawn and aggressive children. Merrill-Palmer Quarterly, 39, 518-534.

Rubin, K. H. \& Mills, R. S. L. (1988). The many faces of social isolation in childhood. Journal of Consulting and Clinical Psychology, 56, 916 - 924. https://doi.org/10.1037/0022-006x.56.6.916

Rubin, K. H., Wojslawowicz, J. C., Rose-Krasnor, L., Booth-LaForce, C. \& Burgess, K. B. (2006). The best friendships of shy/withdrawn children. Prevalence, stability, and relationship quality. Journal of Abnormal Child Psychology, 34, 143-157. https://doi. org/10.1007/s10802-005-9017-4

Schneider, S. \& In-Albon, T. (2010). Angststörungen und Phobien im Kindes- und Jugendalter. Psychotherapeut, 55, 525-540. https://doi.org/10.1007/s00278-010-0724-0

Strauss, C. C., Lahey, B. B., Frick, P., Frame, C. L. \& Hynd, G. W. (1988). Peer social status of children with anxiety disorders. Journal of Consulting and Clinical Psychology, 56, 137-141. https://doi. org/10.1037/0022-006X.56.1.137

Verduin, T. L. \& Kendall, P. C. (2008). Peer perceptions and liking of children with anxiety disorders. Journal of Abnormal Child Psychology, 36, 459 - 469. https://doi.org/10.1007/s10802-007-9192-6

Weber, S. \& Huber, C. (2020). Förderung sozialer Integration durch Kooperatives Lernen - Ein systematisches Review. Empirische Sonderpädagogik, 12, $257-278$.

Weitkamp, K., Romer, G., Rosenthal, S., Wiegand-Grefe, S. \& Daniels, J. (2010). German Screen for Child Anxiety Related Emotional Disorders (SCARED): Reliability, Validity, and Cross-Informant Agreement in a Clinical Sample. Child and Adolescent Psychiatry and Mental Health, 4, 19. https://doi.org/10.1186/ 1753-2000-4-19

World Health Organization. (2016). International statistical classification of diseases and related health problems. ICD-10 (10th revision, 5th ed.). Geneva: World Health Organization.

\section{Historie}

Manuskript eingereicht: 29.09.2020

Manuskript nach Revision angenommen: 10.03.2021

Onlineveröffentlichung: 30.03.2021

\section{Förderung}

Open-Access-Veröffentlichung ermöglicht durch die Bergische Universität Wuppertal.

\section{ORCID}

Simone Weber

(i) https://orcid.org/0000-0003-2533-0666

\section{Simone Weber}

Bergische Universität Wuppertal

School of Education

Institut für Bildungsforschung

Gaußstraße 20

42119 Wuppertal

siweber@uni-wuppertal.de 